durchschlägt, weshalb auch Erdmann ihre ärztlichen Kollegen ermuntert, KHK-Familien den Genomforschern vorzustellen. Der Herzinfarkt kann also doch manchmal eine „beinahe monogene Erkrankung“ sein, wenngleich nur außerordentlich selten - eine Rarität.

\section{Wann werden}

\section{Risikogene klinisch relevant?}

Einzelne Risikovarianten haben allerdings das Zeug, therapeutische Ansatzpunkte zu liefern, wie ADAMTS7, das in genomweiten Assoziationsstudien gefunden und von Wissenschaftlern isoliert und definiert wurde. Mehr noch: Es konnten Mausstämme gezüchtet werden, denen die ADAMTS7-Variante fehlt. Die Experimente ließen den Schluss zu, dass die Inhibition von ADAMTS7 Restenosen verhindern könnte. Erdmann zeigte am Beispiel dieser Variante, wie sehr

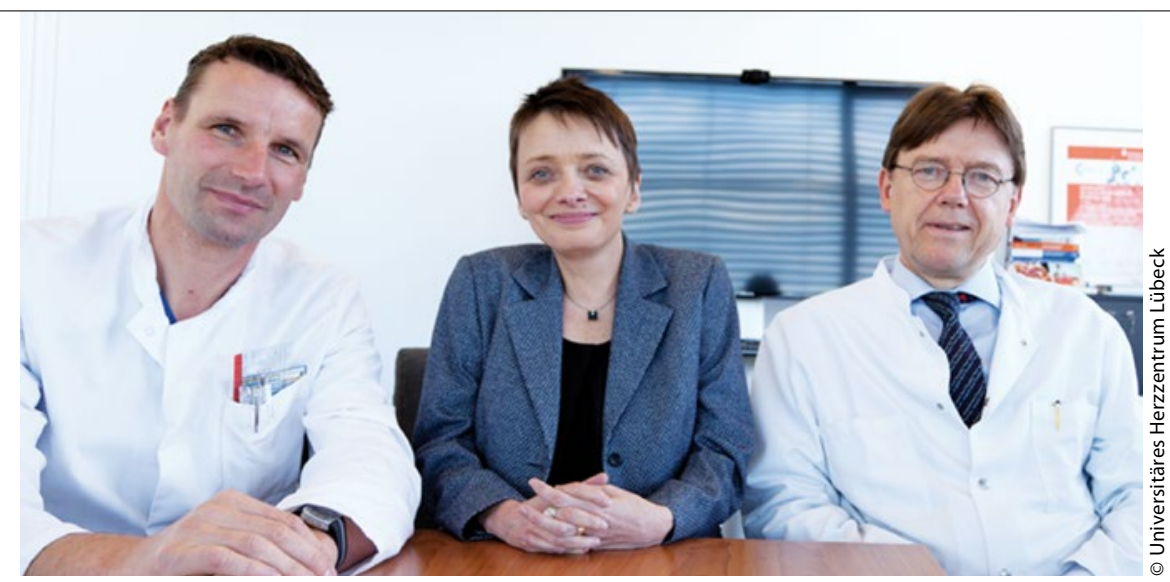

Gründungsmitglieder des Universitären Herzzentrums Lübeck (von links nach rechts: Prof. Holger Thiele, Prof. Jeanette Erdmann, Prof. Hans-Hinrich Sievers).

Umwelt und Gene interagieren: Bei Rauchern mit ADAMTS7 steigt das Herzinfarktrisiko besonders stark.

Die Genomforschung nähert sich zusehends der praktischen Anwendung. Schon können genetische Risikoscores auf dem Boden von SNPs erstellt werden, mithilfe sogenannter Gen-Chips. Noch ist aber die Aussagekraft solcher ChipResultate nicht validiert. Bringen sie mehr als die Familienanamnese? Sollte die Genomanalyse künftig Teil der Risikostratefizierung sein? Viele Fragen sind noch offen, die Genomforschung ist also noch lange nicht ausgereizt.

Dr. med. Jochen Aumiller

\section{Macht die CT-Angiografie die invasive Diagnostik überflüssig?}

Die CT-Koronarangiografie kann "negative Katheter" ersetzen. Nach Ausschluss von Koronarstenosen mittels CT ist die Ereignisrate "nahe Null", und zwar bei Patienten mit stabiler Angina pectoris oder akutem Thoraxschmerz. So beantwortete jedenfalls Prof. Stephan Achenbach, Erlangen, die Frage: Macht die CT-Angiografie die invasive Diagnostik überflüssig?

$F_{\mathrm{d}}^{\mathrm{u}}$ ür die These, dass die CT-Angiografie die invasive Diagnostik überflüssig macht, spricht vor allem die hohe Sensitivität des CTs; vorausgesetzt, es kommen mindestens 64-Zeiler zum Einsatz. In einer aktuellen Metaanalyse, die 30 Studien mit 3.722 Patienten einschloss, ergab sich eine Sensitivität von 95,6\% (95-99\%).

Im Vergleich dazu die Sensitivität anderer nicht-invasiver Tests zur Diagnose einer KHK: Belastungs-EKG: 45-50\%, Belastungs-Stress-Echo: 80-85\%, Belastungs-Stress-SPECT: 73-92\%, Dobutamin-Stress-Echo: 79-83\%, Dobutamin Stress-MRT: $79-88 \%$, Stress-MRT mit Vasodilatator: 67-94\%.

Die unerreicht hohe Sensitivität ist also ein Garant für eine zuverlässige Stenosedetektion. In den ESC-Leitlinien von 2015 wird deshalb die CT-Angiogra- fie als Alternative zur invasiven Koronarangiografie aufgeführt, um ein akutes Koronarsyndrom auszuschließen, sofern die Vortestwahrscheinlichkeit niedrig oder mittelgradig ist und das kardiale Troponin und/oder das EKG nicht zweifelsfrei ausfallen.

Prof. Stephan Achenbach aus Erlangen demonstrierte, dass es auch unter dem CT falsch-positive Ergebnisse gibt, wenn die Bildqualität schlecht ist. Diese können durch veraltete Geräte bedingt sein, aber auch durch mangelhafte Vorbereitung: Es müssen Nitrate gegeben und die Atemkommandos eingehalten werden, die Herzfrequenz sollte möglichst unter 70 Schläge/Minute eingestellt sein. Überhaupt nicht geeignet für das CT sind Patienten mit Vorhofflimmern und starkem Übergewicht. Ferner müssen die Patienten in der Lage sein, die Atemkommandos zu verstehen und auszuführen.

\section{Vernünftige Strahlendosen bei guter Qualität}

Nach Ausschluss einer KHK mithilfe des CTs ist nach Achenbach keine weitere Diagnostik, auch keine spezifische Medikation mehr nötig; die Patienten können beruhigt und entlassen werden. Er beruft sich hierbei auf die CONFIRM-Studie (J Am Coll Cardiac 2011; 58(8):849), eine internationale Multicenterstudie mit knapp 24.000 Personen mit Verdacht auf eine KHK.

In der Diskussion ist nach wie vor die Strahlenbelastung im Rahmen der CTDiagnostik. Nach Achenbach sind heute „vernünftige Dosen“ möglich. Im Deutschen Herz-CT-Register konnten 7.061 CTs ausgewertet werden. Die mittlere Dosis betrug 3,6 mSv. Mit neueren Geräten und Schutzvorkehrungen sind heute CTs mit unter $1 \mathrm{mSv}$ bei ausreichend guter Qualität zu machen.

Um Missverständnisse zu vermeiden: Die Rede ist hierbei von der CT-Angiografie zum Ausschluss einer Koronarstenose, nicht von der CT zum Nachweis von Koronarkalk (Agata-Score), dazu sind deutlich weniger Strahlen nötig. 